\title{
Operando Liquid Pressure Determination in Polymer Electrolyte Fuel Cells
}

\author{
Adrian Mularczyk, Qingyang Lin,* Daniel Niblett, Alexandru Vasile, Martin J. Blunt, Vahid Niasar, \\ Federica Marone, Thomas J. Schmidt, Felix N. Büchi, and Jens Eller*
}

Cite This: ACS Appl. Mater. Interfaces 2021, 13, 34003-34011

Read Online

\section{ACCESS | Lill Metrics \& More | 回 Article Recommendations ｜（） Supporting Information}

ABSTRACT: Extending the operating range of fuel cells to higher current densities is limited by the ability of the cell to remove the water produced by the electrochemical reaction, avoiding flooding of the gas diffusion layers. It is therefore of great interest to understand the complex and dynamic mechanisms of water cluster formation in an operando fuel cell setting as this can elucidate necessary changes to the gas diffusion layer properties with the goal of minimizing the number, size, and instability of the water clusters formed. In this study, we investigate the cluster formation process using X-ray tomographic microscopy at $1 \mathrm{~Hz}$ frequency combined with interfacial curvature analysis and volume-of-fluid simulations to assess the pressure evolution in the water phase. This made it possible to observe the increase in capillary pressure when the advancing water front had to overcome a throat between two neighboring pores and the nuanced interactions of volume and pressure evolution during the droplet formation and its feeding path instability. A $2 \mathrm{kPa}$ higher breakthrough pressure compared to static ex situ capillary pressure versus saturation

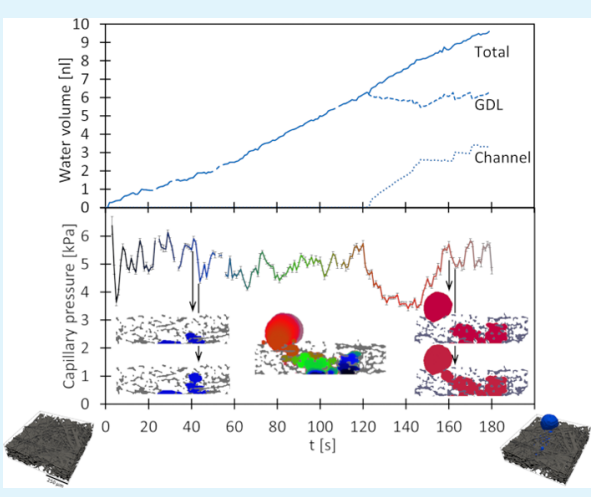
evaluations was observed, which suggests a rethinking of the dynamic liquid water invasion process in polymer electrolyte fuel cell gas diffusion layers.

KEYWORDS: operando, percolation network, fuel cell, water, droplet, pressure, XTM

\section{INTRODUCTION}

In polymer electrolyte fuel cells (PEFCs), electricity is produced from the electrochemical combination of hydrogen and oxygen gas with the formation of water. The water generation occurs in the cathode catalyst layer, and, depending on the operating conditions of the cell, liquid water clusters grow and expand into the neighboring gas diffusion layer (GDL). Within the GDL made from carbon fibers, the water occupies the pore spaces, which are vital to supply gas to the catalyst layer. The fuel cell performance decreases when the pores get blocked by the liquid water generated (flooding). ${ }^{1,2}$ This phenomenon commonly occurs at high current densities, as the water generation rate and current density are intrinsically linked to the electrochemical reaction; increasing the current density increases the amount of water generated. However, high current density operation is desirable in many scenarios as it offers higher specific power density of the cell. Therefore, it is essential to optimize the water management in the GDL, by either avoiding the accumulation of liquid water or managing the water produced in such a way that it does not limit the gas transport to the catalyst layer. ${ }^{3-5}$

In this study, we investigate the mechanisms of water accumulation to improve water management strategies in fuel cells. This understanding is facilitated by imaging techniques sensitive to water such as neutron ${ }^{6-8}$ and X-ray imaging. ${ }^{9-14}$
X-ray tomographic microscopy (XTM) specifically has shown great promise in small-scale studies focusing on interactions between the water and the GDL structure in the micrometer range and with increasingly higher temporal resolution. Recent developments in synchrotron-based XTM at the TOMCAT beamline of the Swiss Light Source (SLS) enable the observation of time-resolved (subsecond time resolution), dynamic processes in $3 \mathrm{D}$ occurring during cluster formation in operando fuel cells, while still maintaining a spatial resolution in the micrometer range. ${ }^{15,16}$

Water progression through the GDL is dominated by capillary forces exerting pressure boundaries on the advancing water front, making the pressure in the water phase a key parameter when trying to describe water cluster formation. Due to the unpredictable nature of the formation of individual water clusters in operando cells, it is difficult to investigate single, independent water clusters from initial formation until the moment of breakthrough into the gas channel. Effects such

Received: March 17, 2021

Accepted: June 23, 2021

Published: July 8, 2021 
as cluster merging have been shown to occur during fuel cell operation as a result of flooding conditions. ${ }^{17}$ Furthermore, it is impossible to determine the rate or continuity of water supplied to a single cluster via the electrochemical reaction when a large electrochemically active area with multiple water clusters is evaluated. The current is unevenly distributed over the active area, and the exact catalyst area contributing to a single cluster is not known and may change over time. ${ }^{18-21}$ In this study, based on the cell developed in earlier work, ${ }^{22,23}$ we developed an XTM compatible operando fuel cell with an active area size of only $200 \mu \mathrm{m}$ by $250 \mu \mathrm{m}$ to encourage the formation of a single water cluster, centered under the gas channel.

While the capillary pressure can be measured in ex situ experiments using water injection into GDLs in the presence of a pressure sensor, ${ }^{24-29}$ it is challenging to obtain the in situ capillary pressure during water formation. Furthermore, common ex situ approaches probe large areas of the GDL with a connected waterfront, resulting in a nonrepresentative characterization as this situation does not occur during regular cell operation where the water instead forms smaller individual clusters. In this study, we determine the pressure in the water phase of a single small cluster from operando XTM imaging at $1 \mathrm{~Hz}$ (one scan acquired every second). Using the segmented XTM data, first, the volumetric evolution of the cluster is revealed, allowing for detailed observations of dynamic processes, otherwise inaccessible under these conditions. The capillary pressure evolution in the water phase is then analyzed using both curvature analysis ${ }^{23,30,31}$ of the segmented water structures and a predictive volume of fluid (VOF) simulation ${ }^{32}$ based on the segmented fiber structure.

\section{METHODS}

Operando PEFC Apparatus. Figure 1a shows the main components of the cell assembly with the two flow fields, the GDLs a)

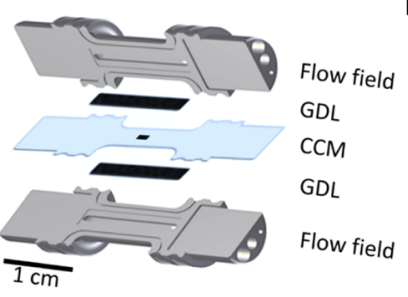

b)

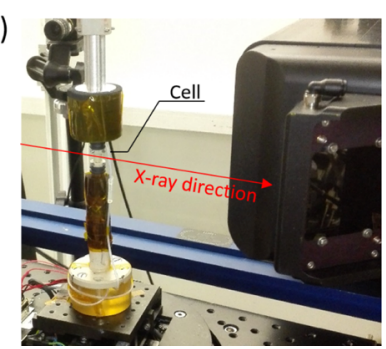

Figure 1. (a) Components making up the imaging cell, used for cluster analysis and (b) cell mounted at the TOMCAT beamline with the red arrow indicating the path of the X-ray beam through the cell to the scintillator at the microscope.

and the catalyst-coated membrane (CCM). Note that the set of sealing gaskets placed around each GDL to prevent gas leakage and control the compression of the GDLs and the compression device keeping the cell assembly together are not shown in this depiction for clarity. Two single-channel flow fields are made of graphite (BMA5, Eisenhuth, Germany), form the anode and cathode sides of the cell. The channels were $800 \mu \mathrm{m}$ wide and $300 \mu \mathrm{m}$ deep with a length of $\sim 14 \mathrm{~mm}$ and feature a rib section on either side of the channel. The GDL material used was a Toray TGP-H 060 with 10 wt \% polytetrafluoroethylene (PTFE) loading. While state-of-the-art GDLs feature an additional microporous layer (MPL), we used a GDL without this MPL for this study since we cannot resolve the pore space in the MPL with the resolution of the XTM and, in any event, we aim to investigate the effects on the GDL domain. The presence of an MPL would definitively have an effect on the water saturation in the GDL, and it is not trivial to anticipate how the water injection mechanism at the GDL-MPL interface would differ to the mechanism at the CL-GDL interface. However, as soon as an initial small water cluster is formed in the GDL domain, its development is controlled by capillary forces-independent of the presence of an MPL in the cell. The CCM was a $15 \mu \mathrm{m}$-thick Gore Primea membrane with 0.1 and $0.4 \mathrm{mg} / \mathrm{cm}^{2} \mathrm{Pt}$ loading on the anode and cathode, respectively. To precisely control the location where the water cluster would form, the catalyst layers on both sides of the CCM were removed by laser ablation, leaving only a 200 by $250 \mu \mathrm{m}^{2}$ active area. This small active area also reduces the probability of forming multiple water clusters that might interact in unintended ways. Previous studies of this material indicate that an active area of this size has a high probability of producing only one fully connected water cluster. ${ }^{10,17}$ The cell was operated at $32{ }^{\circ} \mathrm{C}$, with fully humidified hydrogen and oxygen, supplied at a speed of $6 \mathrm{~m} / \mathrm{s}$ to anode and cathode gas flow channels, respectively. While the temperature was chosen according to experimental limitations, the gas velocity aims to emulate conditions found in large cells of which only a differential section is analyzed here.

XTM Data Acquisition. The cell was mounted in a sample holder at the TOMCAT beamline of the SLS where the experiments were performed, shown in Figure 1b. The full X-ray beam emitted by the $2.9 \mathrm{~T}$ bending magnet was filtered using $20 \mathrm{~mm}$ of glassy carbon (Sigradur, HTW Hochtemperatur-Werkstoffe GmbH, Germany) and $75 \mu \mathrm{m}$ Mo to about $5 \%$ of the original power and was converted with a $150 \mu \mathrm{m} \mathrm{LuAg} / \mathrm{Ce}$ scintillator to visible light and then magnified using a high numerical aperture $4 \times$ optical microscope (Optique Peter). ${ }^{15}$ Images were acquired with the GigaFRoST camera. ${ }^{33}$ The obtained radiographies were tomographically reconstructed ${ }^{34,35}$ to obtain the 3D information of the internal structure with a voxel edge length of $2.75 \mu \mathrm{m}$ at a scan time of $250 \mathrm{~ms}$. The interval between the start of two scans was $1 \mathrm{~s}$. To facilitate this high image acquisition frequency, the cell needs to be rotated continuously for the entire duration of the scanning procedure (180 s) and a combined slip ring and rotary union was used for electrical connections, sensors, and gas supply to the cell. To capture the full process of water cluster formation, the previously dried cell was subjected to constant current operation at $0.62 \mathrm{~mA}\left(\sim 1.2 \mathrm{~A} / \mathrm{cm}^{2}\right)$, starting with the scanning sequence of 180 scans. A full list of the image XTM parameters is given in Table 1.

Table 1. Image Acquisition Parameters Used during Cell Operation (Wet Scan) and for the Reference Scan of the Dried Structure (Dry Scan)

\begin{tabular}{lll}
\multicolumn{1}{c}{ parameter } & wet scan & dry scan \\
number of projections & 301 & 1001 \\
exposure time per projection & $0.83 \mathrm{~ms}$ & $0.83 \mathrm{~ms}$ \\
total scan time & $250 \mathrm{~ms}$ & $831 \mathrm{~ms}$ \\
scan frequency & $1 \mathrm{~Hz}$ & \\
number of scans & 180 & 1 \\
\hline
\end{tabular}

Image Processing. The water phase was segmented from the difference image between the wet and dry scans, while the solid structure is derived from the dry scan only. Details of the image processing and segmentation can be found in Mularczyk et al. ${ }^{23}$ Note that to improve the quality of the dry structure segmentation required for a more accurate VOF simulation, the dry structure was scanned after the experiments at the beamline at half the voxel edge length ( $1.375 \mu \mathrm{m}$ voxel edge length) in a lab micro-CT scanner (Nanotom m, GE Sensing \& Inspection, Wunstorf, Germany).

Curvature Analysis and VOF Simulation. The segmented structures have been analyzed, and the curvature of the gas/water interface was evaluated using a processing method developed for multiphase flow problems in geological samples by Lin et al. ${ }^{31,36}$ The mean interfacial curvature obtained from the images can then be 

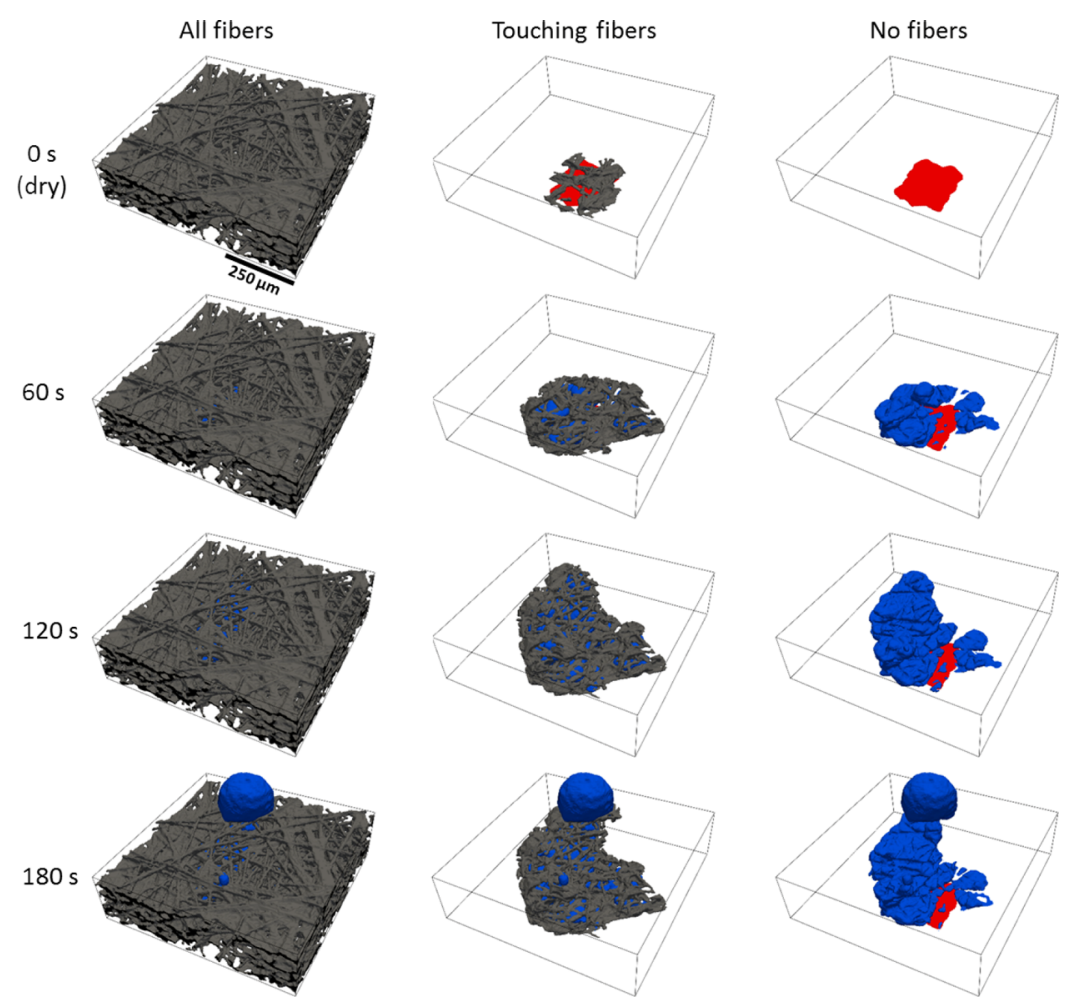

Figure 2. Water cluster growth stages at 0,60,120, and $180 \mathrm{~s}$ after starting of an electrical current. The first column shows all fibers, and the second one shows only the fibers directly surrounding the water cluster and the active area, while the third column shows only the water. The red domain indicates the catalytically active area, while the black box marks the outline of the GDL subsection analyzed.

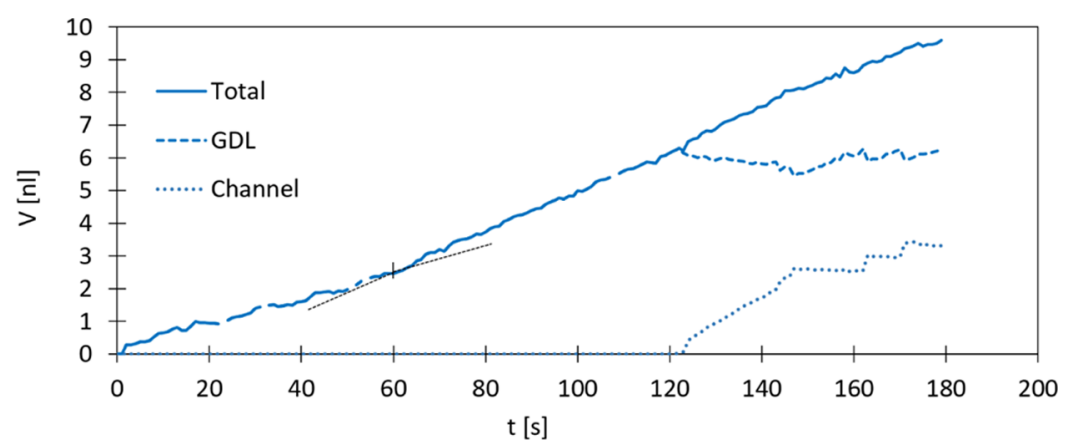

Figure 3. Water volume evolution from the onset of current production for the total system, the channel, and the GDL domain obtained from the segmented XTM data. Blank sections are a result of imaging artifacts preventing the respective time step to be evaluated. Black lines indicate slope change at $60 \mathrm{~s}$.

linked to the pressure in the liquid phase $p_{\mathrm{w}}$ (relative to the assumed constant pressure in the gas) using the Young Laplace equation

$$
p_{\mathrm{w}}=\frac{2 \gamma}{R_{\mathrm{m}}}
$$

where $\gamma$ is the surface tension of the liquid $(71 \mathrm{mN} / \mathrm{m})$ and $R_{\mathrm{m}}$ is the harmonic mean radius of curvature.

More details to the way this method is applied and evaluated for the case of GDL ex situ liquid water injection can be found in Mularczyk et al. $^{23}$

The dry structure segmentation of the GDL obtained with a voxel size of $1.375 \mu \mathrm{m}$ was used as the input for a predictive VOF simulation $^{32,37}$ under the assumption of a constant water-air-GDL contact angle of $156^{\circ}$ (determined using the sessile drop method). The resulting volumetric information about the water structure and the pressure evolution was then compared to the experimentally observed water structure and the pressure derived from the interfacial curvature.

\section{RESULTS AND DISCUSSION}

Characterization of Water Evolution Using XTM. The water cluster formation was imaged at $1 \mathrm{~Hz}$ frequency (1 s intervals) from a state where the entire GDL was dry to past breakthrough of water into the gas channel. Figure 2 shows this process qualitatively in four steps $(60 \mathrm{~s}$ intervals) presenting fibers and water, the subsection of the fiber domain close to water, and the liquid morphology in the GDL. A 3D visualization of the water network evolution including all time steps is provided in Supporting Information.

The first case marked $t=0 \mathrm{~s}$ shows the structure in its completely dry state and corresponds to the dry structure scan taken after acquisition of all wet scans. This state shows the location of the active area (red in Figure 2) and the fiber structure immediately surrounding it. At $t=60 \mathrm{~s}$, the water cluster formation has progressed into the GDL domain. The water has also spread in a lateral direction, exceeding the 
catalytically active domain. A reason for this could be the hydrophilic nature of the membrane in this region. The third row of Figure 2, at $t=120 \mathrm{~s}$, shows the final extent of the water cluster in the GDL domain as it is right before breakthrough into the gas channel at $t=122 \mathrm{~s}$. After breakthrough, a droplet starts forming in the gas channel on the surface of the GDL. Its position and shape can be seen in the final set of images corresponding to $t=180 \mathrm{~s}$. From this, it can be seen that the small active area successfully produces a single water cluster only. Aside from a few small pockets of water on the right side of the catalyst layer, the water forms a single connected network during the observed time period. Note that the small clusters on the right side might be connected to the rest of the water in the domain through pathways close to or inside the catalyst layer; these cannot, however, be reliably resolved due to the small pore size in the catalyst layer and strong imaging artifacts very close to the catalyst layer.

The volumetric evolution of water in the GDL structure was derived from the segmented images by multiplying the number of water voxels $\left(n_{\text {vox }}\right)$ with the voxel volume $\left(V_{\text {vox }}\right)$ of $20.8 \mu \mathrm{m}^{3}$ at each time step using the following equation.

$$
V_{x}=V_{\text {vox }} \times n_{\text {vox }}
$$

where $V_{x}$ denotes the volume of water in the respective domain $x$.

In Figure 3, the resulting volume of water is shown as a function of time for the entire domain and just the GDL and the channel domain.

We observed a linear increase in water volume over the time of the measurement which is in accordance with the constant current operation of the cell during this period. Note that the slight change in slope around the $60 \mathrm{~s}$ mark is associated with evaporation as for the first $60 \mathrm{~s}$, the humidity of the inlet gas was slightly below $\mathrm{RH}=1$. After this point, the slope increases slightly to $\sim 0.06 \mathrm{~nL} / \mathrm{s}$ which is in line with the amount of water generated at a current of $0.62 \mathrm{~mA}$. From Figure 3, it can be observed that the breakthrough of water from the GDL into the gas channel occurs at $t=122 \mathrm{~s}$ when water was observed in the gas channel. After breakthrough, the water volume in the gas channel increases steadily until $t=147 \mathrm{~s}$, while the water volume inside the GDL starts to drop slightly. This means that not only does any newly produced water get fed into the droplet in the channel but also that a small amount of the water accumulated in the GDL drains into the droplet as well. After $t=147 \mathrm{~s}$, it can be seen that the volume in the channel stays unchanged until $t=162 \mathrm{~s}$. During this time, the water volume in the GDL increases again. This is because the water cluster and the droplet get temporarily disconnected between $t$ $=147-162 \mathrm{~s}$. At $t=162 \mathrm{~s}$ and $t=170 \mathrm{~s}$, the volume of the droplet in the channel increases spontaneously, while the water volume in the GDL drops. This is happening due to sporadic connections between the GDL cluster and the droplet at these points in time, causing the droplet in the channel to grow intermittently rather than continuously as was the case initially after breakthrough. These findings are in line with observations made in previous X-ray studies of fuel cells. ${ }^{38,39}$

Figure 4 shows the progression of the water cluster using colors to indicate the temporal evolution, showing the water cluster and droplet formation. The columns of Figure 4 show information from the $2 \mathrm{D}$ slices indicated by the planes in the renderings at the top of each column. This gives a better idea of the way the water moves through the GDL structure with time. Wherever a smooth color gradient is visible, the water

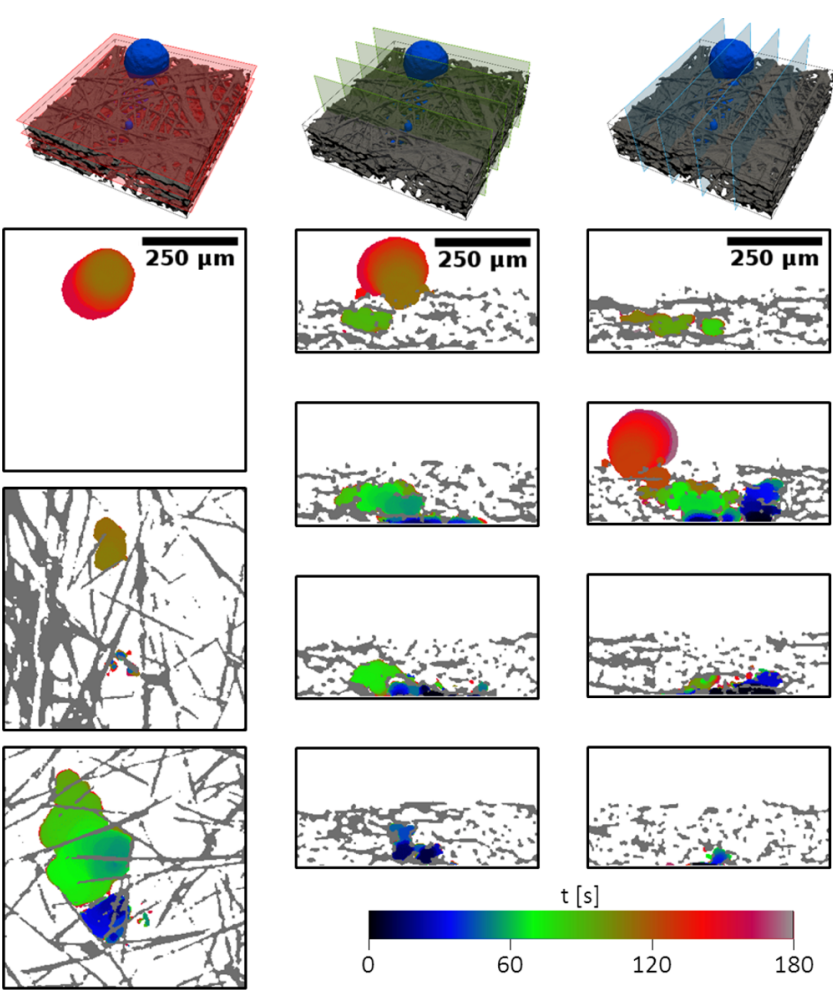

Figure 4. Water cluster growth extracted on cardinal planes through the domain as indicated in the 3D images. The cluster coloring encodes the time at which the water reached the respective location.

cluster grew continuously, and where a sudden jump in color is found, the respective meniscus was halted in its progress for some time before growth continued. This can be seen in the droplet on top of the GDL showing first the continuous filling in the form of a gradual change in color followed by jumps in color as the droplet growth is first halted for some time before spontaneously growing in size. This behavior is also observed in the GDL domain.

Curvature Analysis. The extraction of the radii of curvature from the water structure focused specifically on only the water inside the GDL domain. To prevent the large surface area of the droplet in the channel from having a dominating effect, in this evaluation, it was excluded and the domain investigated was limited to the GDL (indicated by the black GDL bounding box in Figure 2). Because the XTM resolution is not high enough to resolve the precise interfaces between the menisci and the carbon fibers of the GDL, the interfacial surfaces nearer than $3 \mu \mathrm{m}$ to the solid fibers were excluded from the curvature analysis, which is consistent with previous work. ${ }^{23}$ The resultant mean curvature information at each time step was used to estimate the capillary pressure in the water phase $p_{\mathrm{w}}$ using eq 1 . Due to the small amount of water volume present in the field of view during the initial steps, resulting in limited menisci, the discussion is restricted to the capillary pressure information obtained after the water cluster reached a size of $\sim 1 \mathrm{~nL}$. Figure 5a shows the capillary pressure evolution. It can be seen that the pressure in the water phase stays consistently high, while the water cluster fills the GDL and grows in volume (20-120 s). After breakthrough occurs at $t=122 \mathrm{~s}$, the pressure drops from $\sim 5.8$ to $\sim 3.5 \mathrm{kPa}$, as a droplet starts to form in the gas channel and some of the water in the GDL is emptied into the droplet. At the same time as the droplet and the water cluster are disconnected $(t=147$ 
a)

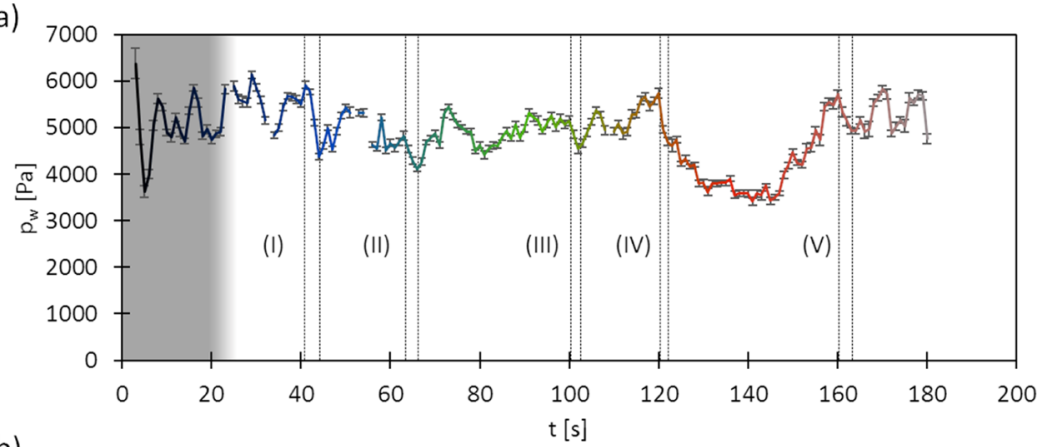

b)
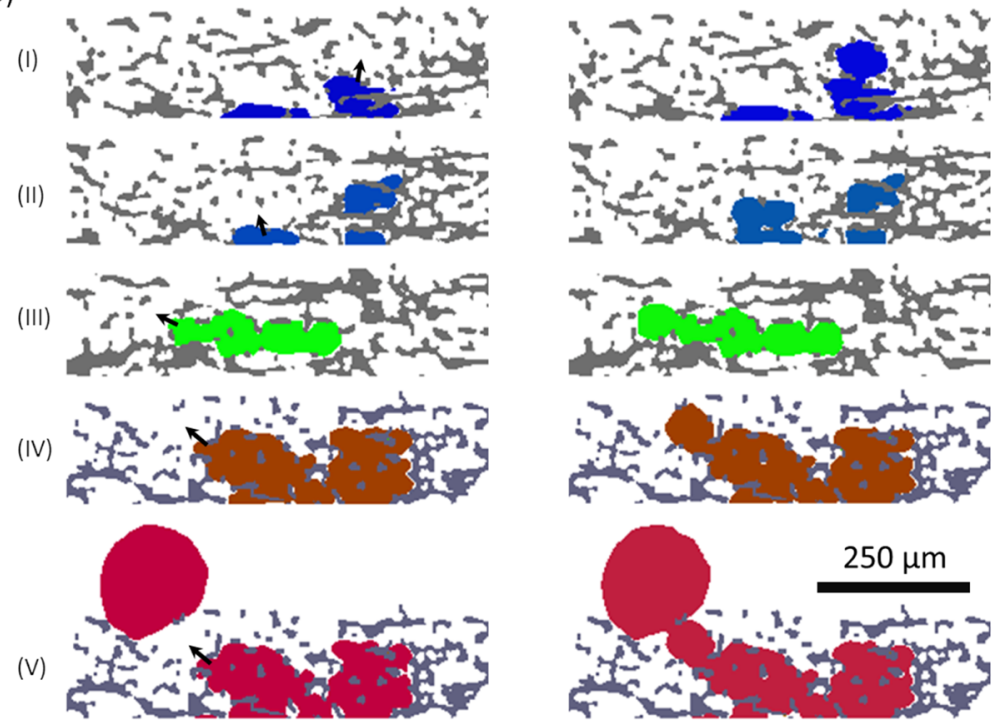

Figure 5. (a) Pressure evolution obtained from curvature analysis. Error bars indicate the standard deviation of the observed surface curvatures at each time step expressed as pressure and (b) pore filling events indicated by the lines (I-V).

s), the pressure can be seen to increase again until the water cluster and the droplet reconnect and the pressure drops again $(t=162 \mathrm{~s})$. This increase and decrease in pressure can be observed for each time the water cluster and droplet disconnect and reconnect, respectively.

Investigating some of the larger pressure fluctuations observed during the filling of the GDL and around the breakthrough and droplet filling events, it was found that the major drops in pressure coincide with breakthrough and pore filling events in the GDL domain. Figure $5 b$ highlights a few of these events by showing the water structure before (left column) and after the pressure drops (right column) indicated with the dashed lines in Figure 5a (I-V). For better visibility, the black arrows in the left column indicate the pore that will be invaded. It is important to note that at the time of initial droplet formation $(\underline{t}=122 \mathrm{~s})$, the drop in pressure precedes the drop in water volume in the GDL domain. This is due to the fact that the "breakthrough pore" is not located directly at the gas channel-GDL interface but instead further inside the GDL domain. The consequence is that the water after breakthrough first fills an intermediate pore in the GDL domain before reaching the gas channel. This also occurs during the intermittent reconnections between the water cluster in the GDL and the droplet in the channel, with the intermittently filled pore emptying when the droplet and GDL water cluster disconnect. This can be seen in Figure 5b (IV and $\mathrm{V}$ ) with the intermittent pore being filled first at pressure drop (IV) and again at pressure drop (V). Note that on the rising edges of the pressure distribution, the water structure was found to increase in size uniformly without expanding into a single pore specifically as is the case when pressure drops are observed.

Predictive VOF Simulation. The water evolution in the GDL domain was simulated at an accelerated rate (to reduce computational time) using the higher resolution dry structure, information about the catalyst area size and location, and a uniform contact angle of $156^{\circ}$ for the entire GDL domain. A visual side-by-side comparison of both the experimentally observed cluster and the results of the simulation is shown shortly after breakthrough in Figure 6a. The VOF simulation provides good qualitative agreement in the overall shape of the water cluster up to the point of breakthrough. The major differences are the absence of two small water clusters observable near the catalyst layer in the experiments (top side in Figure 6a) and the final breakthrough into the gas channel occurring at a different location (black arrows). Due to this difference in the breakthrough location, the comparison of the pressure evolution, Figure $6 b$, is carried out until the moment before breakthrough by normalization of the time using the following equation

$$
t_{\mathrm{norm}}=\frac{t}{t_{\mathrm{BT}}}
$$

where $t$ is the time in seconds and $t_{\mathrm{BT}}$ denotes the time when breakthrough was observed, corresponding to $t_{\mathrm{BT}}=120 \mathrm{~s}$ in the experimental case. 


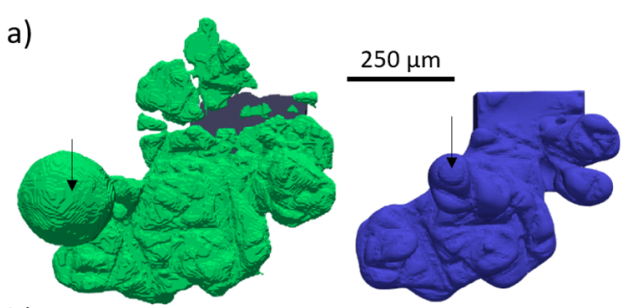

b)

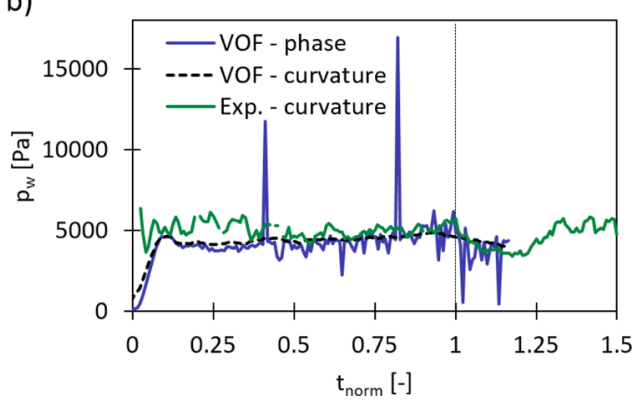

Figure 6. (a) Side-by-side comparison of the water cluster obtained from XTM (green) and the VOF simulation (blue). (b) Pressure evolution obtained from the phase of the VOF results (blue) compared to the pressure obtained from the curvature analysis performed on the VOF results (black) and on the experimental structure (green). The time is the normalized time with the start of growth at 0 and breakthrough at 1 .

The pressure obtained from the VOF simulation first increases rapidly to $\sim 5 \mathrm{kPa}$ after which it slowly increases until breakthrough occurs at a pressure similar to the one obtained from the curvature analysis. There are some fluctuations observed as well; however, these stem mostly from numerical instabilities. Note that the average $p_{\mathrm{w}}$ values resulted from VOF simulations have been directly calculated from the phase pressure, while $p_{\mathrm{w}}$ values from the experiments have been indirectly estimated from eq 1 . Furthermore, due to the fact that the VOF simulation does not take into account contact angle hysteresis between advancing and receding contact angles, cluster shrinking events caused by events such as the formation of the droplet on the GDL are not accurately represented as the structure tends to contract a lot more than would be the case otherwise. As such, it is difficult to draw further conclusions from the comparison of the XTM observations and VOF simulation results past breakthrough. The capillary pressure evaluation based on the radius of curvature was also computed on the interfaces obtained by the VOF simulation results under the assumption of the pressure being in equilibrium at each time step and included in Figure 6b. While the resulting capillary pressure is valid on average, it does not capture the fluctuations in the volume-averaged phase pressures obtained by the VOF simulation.

However, given the simplifications used in the VOF simulation, the results give further indication that the small clusters adjacent to the active area, observed in the XTM data but absent in the VOF simulation, are likely due to the water connections in the catalyst layer or near the hydrophilic membrane, which is not captured by the simulation.

Capillary Pressure Evolution and Breakthrough Pressure. In this study, it was observed that both the curvature analysis and the VOF simulation indicate a rapid capillary pressure increase already for small amounts of water in the GDL. The pressure remains at a high level, while the percolation network develops in the GDL and increases in size.
In fact, pressures close to the final breakthrough pressure are observed, while the cluster is expanding through the GDL domain. This behavior is different from ex situ liquid water injection-based determinations of capillary pressure versus saturation data for this material (TGP-H $06010 \mathrm{wt} \%$ ) on large representative domains. ${ }^{25,27}$ In these types of measurements, the saturation commonly increases gradually with capillary pressure until breakthrough is reached. Furthermore, the final breakthrough pressure $p_{\mathrm{BT}}$ of $5.7 \mathrm{kPa}$, observed in this study, is above the values found in the literature for ex situ injection experiments of $\sim 3.5-5.4 \mathrm{kPa}$. However, both differences can be explained when considering the difference in the probed GDL area. Looking at the published breakthrough pressure values against the injection area used in the respective publications, there is the trend that the smaller the area of the GDL exposed to the injected liquid water, the higher the observed breakthrough pressure (Figure 7). This is

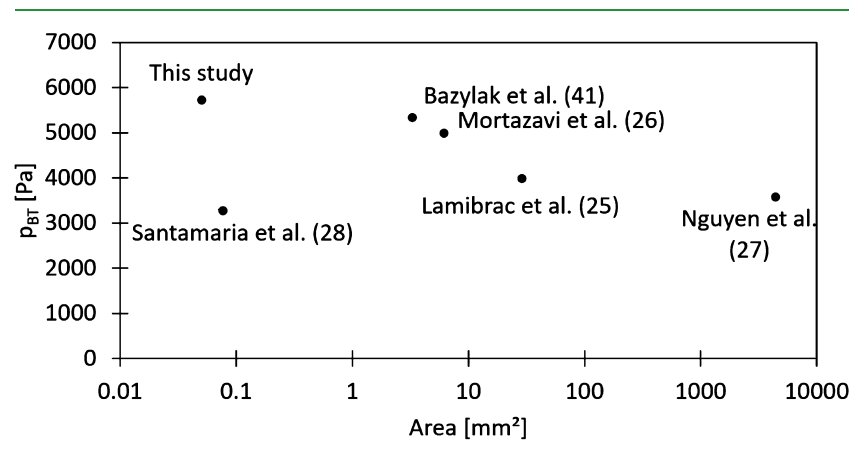

Figure 7. Breakthrough pressure vs area of the GDL exposed to the water during the experiments for Toray TGP-H-060 (10 wt \% PTFE). Data taken from studies of the same material. ${ }^{25-28,41}$

due to the pore size of the GDL not being uniform but rather a distribution, ${ }^{25}$ which means that statistically, the larger the exposed GDL domain, the higher the likelihood for the connected water to encounter a pathway with many large pores, requiring less pressure to reach breakthrough. In contrast, smaller domains contain only a limited number of pores with a smaller likelihood of there being a percolating pathway of larger-than-average pores. Therefore, these domains exhibit a higher breakthrough pressure. This effect has also been previously described by Santamaria et al. and Nam et al. ${ }^{28,40}$ There is, however, still a chance to hit a percolation path with large pores and throats even with small injection geometries which could explain the low values observed by Santamaria et al. ${ }^{28}$ for this material.

\section{CONCLUSIONS}

Using XTM at the Tomcat beamline of the SLS, it was possible to investigate the formation of a single water cluster in an operando imaging cell. The percolation cluster evolution was imaged with a frequency of $1 \mathrm{~Hz}$ and a voxel size of $2.75 \mu \mathrm{m}$. This imaging gives a unique insight into dynamic interactions of the water and the GDL.

While the rate of cluster growth stayed mostly constant during filling of the GDL, the volumetric analysis revealed the presence of two distinct modes of droplet filling once breakthrough occurs. First, a continuous feeding is observed, where the water cluster in the GDL and the droplet in the channel remained connected, maintaining an approximately constant water volume in the GDL. This steady state can 
change when the water in the GDL disconnects from the channel, and we have intermittent feeding during which the water cluster in the GDL accumulates water and swells before sporadically and repeatedly emptying into the water droplet in the channel.

Capillary pressure evaluation via interfacial curvature analysis revealed that both feeding mechanisms are correlated to significant changes in pressure in the water phase of approximately $2 \mathrm{kPa}$ for the continuous feed and $0.9 \mathrm{kPa}$ for the intermittent feed. Furthermore, while the rate of change of water volume in the GDL during the GDL filling was observed to be very constant, the curvature analysis shows the presence of pressure fluctuations during cluster formation which were found to correspond to the breakthrough through narrower regions of the pore space and pore filling events, highlighting the large number of pressure fluctuations experienced by the percolation cluster before even reaching the gas channel. This demonstrates the extraordinary capabilities of this analysis to disseminate effects and interactions previously unobtainable in operando fuel cell investigations. Furthermore, these fluctuations could cause unwanted water propagation in the in-plane direction due to the in-plane pores being commonly larger than the through-plane pores for this type of material. ${ }^{25}$ The resulting menisci deformation in the in-plane direction would, on average, be larger as well and could be a source for lateral water movement and cluster merging.

The VOF simulation results show good agreement with the water structure obtained experimentally despite limited knowledge about local contact angles and coating distribution inside the GDL domain. Furthermore, the pressure evolution before breakthrough agrees well with the consistently high pressures observed with the curvature analysis.

Both the curvature analysis and simulation indicate that the pressure needed to progress the evolution of water though the GDL domain is consistently high and similar to the breakthrough pressure, which was found to be higher than previously published values. This contradicts the behavior expected from measured static capillary pressure versus saturation functions. The authors attribute this to the fact that these studies are commonly performed on larger GDL samples to obtain averaged results independent of the inhomogeneous nature of the material. This, however, exposes the water to many more pores and throats and thus increases the probability of GDL filling and breakthrough to occur already at lower pressures as the water will inevitably take the path of the least resistance.

Contrary to this, the current study investigated the strongly localized environment encountered by a typical water cluster in a running fuel cell. While this does not exclude the probability of clusters with low breakthrough pressure to form, this observation highlights the fact that in many cases, higher pressure values are to be expected for noncommunicating clusters over large fuel cell areas, than would be observed using large-area, ex situ investigations, and need to be considered, that is, in predictive two-phase PEFC simulations.

\section{ASSOCIATED CONTENT}

\section{SI Supporting Information}

The Supporting Information is available free of charge at https://pubs.acs.org/doi/10.1021/acsami.1c04560.
Droplet growth over $180 \mathrm{~s}$ indicating the gas channel and the rib sections of the cell using black wireframes (AVI)

\section{AUTHOR INFORMATION}

\section{Corresponding Authors}

Qingyang Lin - State Key Laboratory of Clean Energy Utilization, State Environmental Protection Engineering Center for Coal-Fired Air Pollution Control, Zhejiang University, Hangzhou 310027, China; 이이.org/00000001-5691-9532; Email: qingyang_lin@zju.edu.cn

Jens Eller - Electrochemistry Laboratory, Paul Scherrer Institut (PSI), Villigen 5232, Switzerland; 이이.org/ 0000-0002-9348-984X; Email: jens.eller@psi.ch

\section{Authors}

Adrian Mularczyk - Electrochemistry Laboratory, Paul Scherrer Institut (PSI), Villigen 5232, Switzerland; (1) orcid.org/0000-0001-7919-3324

Daniel Niblett - Department of Chemical Engineering and Analytical Science, University of Manchester, Manchester M13 9LP, U.K.

Alexandru Vasile - Electrochemistry Laboratory, Paul Scherrer Institut (PSI), Villigen 5232, Switzerland

Martin J. Blunt - Department of Earth Science and Engineering, Imperial College London, London SW7 2AZ, U.K.

Vahid Niasar - Department of Chemical Engineering and Analytical Science, University of Manchester, Manchester M13 9LP, U.K.; (1) orcid.org/0000-0002-9472-555X

Federica Marone - Swiss Light Source, Paul Scherrer Institut (PSI), Villigen 5232, Switzerland

Thomas J. Schmidt - Electrochemistry Laboratory, Paul Scherrer Institut (PSI), Villigen 5232, Switzerland; Laboratory of Physical Chemistry, ETH Zürich, Zürich 8093,

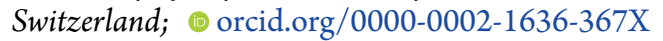

Felix N. Büchi - Electrochemistry Laboratory, Paul Scherrer Institut (PSI), Villigen 5232, Switzerland; (1) orcid.org/ 0000-0002-3541-4591

Complete contact information is available at:

https://pubs.acs.org/10.1021/acsami.1c04560

\section{Author Contributions}

The manuscript was written by A.M. with support from F.N.B., J.E., and T.J.S. A.M. conducted the experimental work and was supported in processing the data by A.V. F.M. supported the image acquisition at the TOMCAT beamline. Q.L. and M.B. performed the analysis of the interface curvature, and D.N. and V.N. carried out the VOF simulations and analysis. All authors contributed to the manuscript revision and have given approval to the final version of the manuscript.

\section{Funding}

The authors gratefully acknowledge the Swiss National Science Foundation (SNSF project no. 169913) and UK Engineering and Physical Sciences Research Council (EPSRC project no. 1930966) for financial support for this research.

\section{Notes}

The authors declare no competing financial interest.

\section{ACKNOWLEDGMENTS}

The authors gratefully acknowledge the support of Hong Xu, Christoph Csoklich, Michael Striednig, and Arnaud Schuller 
during the acquisition of the XTM images. Thomas Gloor and Marcel Hottiger are acknowledged for their support with setup development and software implementations. We acknowledge the Paul Scherrer Institut, Villigen, Switzerland, for provision of synchrotron radiation beamtime at the TOMCAT beamline of the SLS.

\section{REFERENCES}

(1) Ihonen, J.; Mikkola, M.; Lindbergh, G. Flooding of Gas Diffusion Backing in Pefcs. J. Electrochem. Soc. 2004, 151, A1152.

(2) Shen, J.; Xu, L.; Chang, H.; Tu, Z.; Chan, S. H. Partial Flooding and Its Effect on the Performance of a Proton Exchange Membrane Fuel Cell. Energy Convers. Manage. 2020, 207, 112537.

(3) Sinha, P. K.; Mukherjee, P. P.; Wang, C.-Y. Impact of Gdl Structure and Wettability on Water Management in Polymer Electrolyte Fuel Cells. J. Mater. Chem. 2007, 17, 3089-3103.

(4) Biesdorf, J.; Forner-Cuenca, A.; Schmidt, T. J.; Boillat, P. Impact of Hydrophobic Coating on Mass Transport Losses in Pefcs. J. Electrochem. Soc. 2015, 162, F1243-F1252.

(5) Tranter, T. G.; Boillat, P.; Mularczyk, A.; Manzi-Orezzoli, V.; Shearing, P. R.; Brett, D. J. L.; Eller, J.; Gostick, J. T.; Forner-Cuenca, A. Pore Network Modelling of Capillary Transport and Relative Diffusivity in Gas Diffusion Layers with Patterned Wettability. J. Electrochem. Soc. 2020, 167, 114512.

(6) Bellows, R. J.; Lin, M. Y.; Arif, M.; Thompson, A. K.; Jacobson, D. Neutron Imaging Technique for in Situ Measurement of Water Transport Gradients within Nafion in Polymer Electrolyte Fuel Cells. J. Electrochem. Soc. 1999, 146, 1099.

(7) Manke, I.; Hartnig, C.; Grünerbel, M.; Kaczerowski, J.; Lehnert, W.; Kardjilov, N.; Hilger, A.; Banhart, J.; Treimer, W.; Strobl, M. Quasi-in Situ Neutron Tomography on Polymer Electrolyte Membrane Fuel Cell Stacks. Appl. Phys. Lett. 2007, 90, 184101.

(8) Owejan, J.; Trabold, T.; Jacobson, D.; Arif, M.; Kandlikar, S. Effects of Flow Field and Diffusion Layer Properties on Water Accumulation in a Pem Fuel Cell. Int. J. Hydrogen Energy 2007, 32, 4489-4502.

(9) Battrell, L.; Patel, V.; Zhu, N.; Zhang, L.; Anderson, R. Imaging of the Desaturation of Gas Diffusion Layers by Synchrotron Computed Tomography. J. Power Sources 2019, 416, 155-162.

(10) Eller, J.; Marone, F.; Büchi, F. N. Operando Sub-Second Tomographic Imaging of Water in Pefc Gas Diffusion Layers. ECS Trans. 2015, 69, 523-531.

(11) Ince, U. U.; Markötter, H.; George, M. G.; Liu, H.; Ge, N.; Lee, J.; Alrwashdeh, S. S.; Zeis, R.; Messerschmidt, M.; Scholta, J.; Bazylak, A.; Manke, I. Effects of Compression on Water Distribution in Gas Diffusion Layer Materials of Pemfc in a Point Injection Device by Means of Synchrotron X-Ray Imaging. Int. J. Hydrogen Energy 2018, 43, 391-406.

(12) Nagai, Y.; Eller, J.; Hatanaka, T.; Yamaguchi, S.; Kato, S.; Kato, A.; Marone, F.; Xu, H.; Büchi, F. N. Improving Water Management in Fuel Cells through Microporous Layer Modifications: Fast Operando Tomographic Imaging of Liquid Water. J. Power Sources 2019, 435, 226809 .

(13) Sasabe, T.; Tsushima, S.; Hirai, S.; Minami, K.; Yada, K. Visualization of Liquid Water Distribution in an Operational Pemfc by Soft X-Ray Radiography. ECS Trans. 2009, 25, 513-521.

(14) Zenyuk, I. V.; Parkinson, D. Y.; Hwang, G.; Weber, A. Z. Probing Water Distribution in Compressed Fuel-Cell Gas-Diffusion Layers Using X-Ray Computed Tomography. Electrochem. Commun. 2015, 53, 24-28.

(15) Bührer, M.; Stampanoni, M.; Rochet, X.; Büchi, F.; Eller, J.; Marone, F. High-Numerical-Aperture Macroscope Optics for TimeResolved Experiments. J. Synchrotron Radiat. 2019, 26, 1161-1172.

(16) Xu, H.; Bührer, M.; Marone, F.; Schmidt, T. J.; Büchi, F. N.; Eller, J. Optimal Image Denoising for in Situ X-Ray Tomographic Microscopy of Liquid Water in Gas Diffusion Layers of Polymer Electrolyte Fuel Cells. J. Electrochem. Soc. 2020, 167, 104505.
(17) Eller, J.; Roth, J.; Marone, F.; Stampanoni, M.; Büchi, F. N. Operando Properties of Gas Diffusion Layers: Saturation and Liquid Permeability. J. Electrochem. Soc. 2016, 164, F115-F126.

(18) Büchi, F. N.; Geiger, A. B.; Neto, R. P. Dependence of Current Distribution on Water Management in Pefc of Technical Size. J. Power Sources 2005, 145, 62-67.

(19) Freunberger, S. A.; Reum, M.; Evertz, J.; Wokaun, A.; Büchi, F. N. Measuring the Current Distribution in Pefcs with Sub-Millimeter Resolution. J. Electrochem. Soc. 2006, 153, A2158.

(20) Liu, Z.; Mao, Z.; Wu, B.; Wang, L.; Schmidt, V. M. Current Density Distribution in Pefc. J. Power Sources 2005, 141, 205-210.

(21) Nishikawa, H.; Kurihara, R.; Sukemori, S.; Sugawara, T.; Kobayasi, H.; Abe, S.; Aoki, T.; Ogami, Y.; Matsunaga, A. Measurements of Humidity and Current Distribution in a Pefc. J. Power Sources 2006, 155, 213-218.

(22) Xu, H.; Bührer, M.; Marone, F.; Schmidt, T. J.; Büchi, F. N.; Eller, J. Fighting the Noise: Towards the Limits of Subsecond X-Ray Tomographic Microscopy of Pefc. ECS Trans. 2017, 80, 395-402.

(23) Mularczyk, A.; Lin, Q.; Blunt, M. J.; Lamibrac, A.; Marone, F.; Schmidt, T. J.; Büchi, F. N.; Eller, J. Droplet and Percolation Network Interactions in a Fuel Cell Gas Diffusion Layer. J. Electrochem. Soc. 2020, 167, 084506.

(24) Bazylak, A.; Sinton, D.; Djilali, N. Dynamic Water Transport and Droplet Emergence in Pemfc Gas Diffusion Layers. J. Power Sources 2008, 176, 240-246.

(25) Lamibrac, A.; Roth, J.; Toulec, M.; Marone, F.; Stampanoni, M.; Büchi, F. N. Characterization of Liquid Water Saturation in Gas Diffusion Layers by X-Ray Tomographic Microscopy. J. Electrochem. Soc. 2015, 163, F202-F209.

(26) Mortazavi, M.; Tajiri, K. Liquid Water Breakthrough Pressure through Gas Diffusion Layer of Proton Exchange Membrane Fuel Cell. Int. J. Hydrogen Energy 2014, 39, 9409-9419.

(27) Nguyen, T. V.; Lin, G.; Ohn, H.; Wang, X. Measurement of Capillary Pressure Property of Gas Diffusion Media Used in Proton Exchange Membrane Fuel Cells. Electrochem. Solid-State Lett. 2008, 11, B127.

(28) Santamaria, A. D.; Das, P. K.; MacDonald, J. C.; Weber, A. Z. Liquid-Water Interactions with Gas-Diffusion-Layer Surfaces. J. Electrochem. Soc. 2014, 161, F1184-F1193.

(29) Quesnel, C.; Cao, R.; Lehr, J.; Kietzig, A.-M.; Weber, A. Z.; Gostick, J. T. Dynamic Percolation and Droplet Growth Behavior in Porous Electrodes of Polymer Electrolyte Fuel Cells. J. Phys. Chem. C 2015, 119, 22934-22944.

(30) Akai, T.; Lin, Q.; Alhosani, A.; Bijeljic, B.; Blunt, M. Quantification of Uncertainty and Best Practice in Computing Interfacial Curvature from Complex Pore Space Images. Materials 2019, 12, 2138

(31) Lin, Q.; Bijeljic, B.; Pini, R.; Blunt, M. J.; Krevor, S. Imaging and Measurement of Pore-Scale Interfacial Curvature to Determine Capillary Pressure Simultaneously with Relative Permeability. Water Resour. Res. 2018, 54, 7046-7060.

(32) Niblett, D.; Mularczyk, A.; Niasar, V.; Eller, J.; Holmes, S. TwoPhase Flow Dynamics in a Gas Diffusion Layer - Gas Channel Microporous Layer System. J. Power Sources 2020, 471, 228427.

(33) Mokso, R.; Schlepütz, C. M.; Theidel, G.; Billich, H.; Schmid, E.; Celcer, T.; Mikuljan, G.; Sala, L.; Marone, F.; Schlumpf, N.; Stampanoni, M. Gigafrost: The Gigabit Fast Readout System for Tomography. J. Synchrotron Radiat. 2017, 24, 1250-1259.

(34) Paganin, D.; Mayo, S. C.; Gureyev, T. E.; Miller, P. R.; Wilkins, S. W. Simultaneous Phase and Amplitude Extraction from a Single Defocused Image of a Homogeneous Object. J. Microsc. 2002, 206, 33-40.

(35) Marone, F.; Stampanoni, M.; Stampanoni, M. F. Regridding Reconstruction Algorithm for Real-Time Tomographic Imaging. J. Synchrotron Radiat. 2012, 19, 1029-1037.

(36) Lin, Q.; Bijeljic, B.; Berg, S.; Pini, R.; Blunt, M. J.; Krevor, S. Minimal Surfaces in Porous Media: Pore-Scale Imaging of Multiphase Flow in an Altered-Wettability Bentheimer Sandstone. Phys. Rev. E 2019, 99, 063105. 
(37) Niblett, D.; Niasar, V.; Holmes, S. Enhancing the Performance of Fuel Cell Gas Diffusion Layers Using Ordered Microstructural Design. J. Electrochem. Soc. 2019, 167, 013520.

(38) Manke, I.; Hartnig, C.; Grünerbel, M.; Lehnert, W.; Kardjilov, N.; Haibel, A.; Hilger, A.; Banhart, J.; Riesemeier, H. Investigation of Water Evolution and Transport in Fuel Cells with High Resolution Synchrotron X-Ray Radiography. Appl. Phys. Lett. 2007, 90, 174105.

(39) Deevanhxay, P.; Sasabe, T.; Tsushima, S.; Hirai, S. Observation of Dynamic Liquid Water Transport in the Microporous Layer and Gas Diffusion Layer of an Operating Pem Fuel Cell by HighResolution Soft X-Ray Radiography. J. Power Sources 2013, 230, 3843.

(40) Nam, J. H.; Lee, K.-J.; Hwang, G.-S.; Kim, C.-J.; Kaviany, M. Microporous Layer for Water Morphology Control in Pemfc. Int. J. Heat Mass Transfer 2009, 52, 2779-2791.

(41) Bazylak, A.; Sinton, D.; Liu, Z.-S.; Djilali, N. Effect of Compression on Liquid Water Transport and Microstructure of Pemfc Gas Diffusion Layers. J. Power Sources 2007, 163, 784-792. 